\section{Reducing the contamination of beef carcasses by specified risk materials during slaughter}

\author{
Daniela Meloni,, ${ }^{1}$ Danilo Pitardi, ${ }^{1}$ \\ Cristiana Maurella, ${ }^{1}$ Dolores Di Vietro, ${ }^{1}$ \\ Luca Nocilla, ${ }^{1}$ Antonio Piscopo, ${ }^{2}$ \\ Elena Pavoletti, ${ }^{3}$ Mauro Negro, ${ }^{4}$ \\ Maria Caramelli, ${ }^{1}$ Elena Bozzetta ${ }^{1}$
}

'Istituto Zooprofilattico Sperimentale del Piemonte, Liguria e Valle d'Aosta, Torino; ${ }^{2}$ Azienda Sanitaria Locale Agrigento, Agrigento; ${ }^{3}$ Azienda Sanitaria Locale Vercelli, Vercelli; ${ }^{4}$ Azienda Sanitaria Locale Mondovì, Mondovì, Italy

\section{Abstract}

According to the European Community Regulation 1139/2003, a sampling plan based on an appropriate tests to detect the presence of central nervous system (CNS) tissue on the surface of the head muscles had to be implemented in each member state starting from 2003. In previous studies, we showed a medium CNS contamination in head meat from Italian slaughterhouses of $14,7 \%$. Recently, German authors referred about alarming rates (82\%) of CNS contamination in para-vertebral muscles after routine carcass splitting and spinal cord removal practices. In that context, the authors intended to assess the percentage of CNS contamination in muscles close to the vertebral column obtained by applying three different removal practices of specific risk material (SRM). Two alternative techniques, by sucking out and by water-jet, were compared to the traditional one; in both situations the removal of spinal cord occurred before the carcass splitting. The percentage of contamination resulted $62 \%$ applying the traditional technique, $60 \%$ with the sucking out system and $36 \%$ using the water-jet. The results show that water-jet ensures the most significant reduction of SRM contamination in para-vertebral meat as it guarantees a rapid and complete extraction of the spinal cord.

\section{Introduzione}

La correlazione emersa fra bovine spongiform encephalopathy (BSE) e nuova variante del morbo di Creuzfeldt Jackob (vCJD) ha evidenziato il carattere zoonosico della malattia e conseguentemente il rischio rappresentato dal consumo di tessuti bovini infetti per l'uomo.

Negli animali colpiti da BSE, il prione si accumula maggiormente in alcuni organi definiti materiale specifico a rischio (MSR), tra i quali il più importante, per concentrazione del patogeno, è il sistema nervoso centrale (SNC), ossia encefalo e midollo spinale.

L'esclusione del MSR dal consumo umano, prevista sin dal 2001 (Commissione Europea, 2001), viene pertanto considerata la misura più efficace ad oggi per ridurre l'esposizione del consumatore al rischio BSE, soprattutto in relazione al progressivo rilassamento delle misure applicate in questo contesto.

Recenti studi hanno evidenziato come le metodiche utilizzate in sede di macellazione per l'allontanamento del MSR determinino importanti contaminazioni da SNC a carico delle carni di spolpo delle teste e delle masse muscolari adiacenti alla colonna vertebrale (Helps et al., 2002; Prendergast et al., 2003; Bowling et al., 2007). Inoltre, le metodiche tradizionali di macellazione mediante divisione della carcassa in mezzene con sega elettrica, prima dell'allontanamento del midollo spinale, sembra possano determinare una aerosolizzazione del MSR con conseguente contaminazione dell'ambiente di macellazione e cross-contaminazione delle carcasse (Troeger, 2004; Ramantanis, 2006).

L'obiettivo è stato quello di comparare l'entità della contaminazione da SNC dei muscoli paravertebrali a seguito di macellazione tradizionale e di applicazione di due tecniche alternative che permettono l'eliminazione del midollo spinale prima della suddivisione della carcassa.

\section{Materiali e Metodi}

\section{Campionamento}

Il campionamento è stato effettuato mediante tampone strofinato su un'area di $10 \times 10 \mathrm{~cm}$ a livello dei muscoli paravertebrali nella regione cervicale. Per ogni animale è stato raccolto un solo campione.

\section{Test Ridascreen risk material 10/5}

Il test è basato su una metodica ELISA con rilevazione immunoenzimatica della proteina acida gliofibrillare (GFAP) presente ad alte concentrazioni a livello del SNC. L'espressione dei risultati è facilitata dall'utilizzo del software RIDA ${ }^{\circledR}$ SOFT Win (R-Biopharm, Darmstadt, Germania) che permette l'elaborazione automatica dei dati e l'espressione dei risultati attraverso retta di regressione.

\section{Prevalenza basale}

Sono state campionate 216 mezzene in un macello di grosse dimensioni $(>4000$ capi/anno) e 196 in uno di medie $(\leq 4000$ capi/anno).
Correspondence: Daniela Meloni, Istituto Zooprofilattico Sperimentale del Piemonte, Liguria e Valle d'Aosta, via Bologna 148, 10154 Torino, Italy.

Tel. +39.011.2686347 - Fax: +39.011.2686322.

E-mail: daniela.meloni@izsto.it

Key words: Specific risk material, Central nervous system, Ridascreen risk material 10/5.

Conflict of interests: the authors declare no potential conflict of interests.

Funding: the work was supported by the Ministry of Health Current Research grant 2008 no. IZSPLV 03/08 RC.

Received for publication: 15 January 2013.

Revision received: 11 February 2013.

Accepted for publication: 11 February 2013.

This work is licensed under a Creative Commons Attribution 3.0 License (by-nc 3.0).

(C) Copyright D. Meloni et al., 2013

Licensee PAGEPress, Italy

Italian Journal of Food Safety 2013; 2:e3

doi:10.4081/ijfs.2013.e3

\section{Studio comparativo}

A seguito dell'applicazione di idrogetto (Piscopo, 2001) e di aria pressurizzata all'interno del canale vertebrale (LGR Equipment, Portile, Italia), e della tecnica tradizionale, 50 campioni sono stati raccolti per ciascuna metodica ( 5 bovini al termine di ogni giornata di macellazione fino al raggiungimento del numero stabilito).

\section{Studio esplorativo}

Lo studio esplorativo è stato eseguito al fine di appurare l'eventuale ruolo della sega circolare come titolare di cross-contaminazione.

\section{Analisi statistica}

$\mathrm{Al}$ fine di determinare la prevalenza di contaminazione con metodica tradizionale e la dimensione del campione, è stata considerata una prevalenza attesa del 15\%, un intervallo di confidenza del $95 \%$ ed un errore accettabile pari al $4 \%$.

La dimensione del campione necessaria per lo studio comparativo è stata ottenuta inserendo i nostri dati in tabelle Kastenbaum-HoelBowman. La distribuzione dei dati è stata valutata mediante un test di normalità basato su asimmetria e curtosi. Poiché i dati ottenuti non sono risultati normalmente distribuiti, è stato eseguito il Kruskal-Wallis equality-ofpopulations rank test per verificare le principali differenze tra i livelli di contaminazione connessi alle tre tecniche di rimozione del MSR. I dati sono stati inseriti in un database e analizzati utilizzando il software Stata $11 \mathrm{SE}$ 
Tabella 1. Risultati dell'analisi statistica effettuata per valutare la differenza fra i tre metodi di rimozione del midollo spinale.

\begin{tabular}{lcc} 
Metodo & Campioni (No.) & Rank sum \\
Tradizionale & 50 & 4407,5 \\
Aspirazione & 50 & 3912 \\
\hline Getto d'acqua & 50 & 3005,5 \\
\hline$\chi^{2}=10,712 ; \mathrm{df}=2 ; \mathrm{P}=0,005$. & &
\end{tabular}

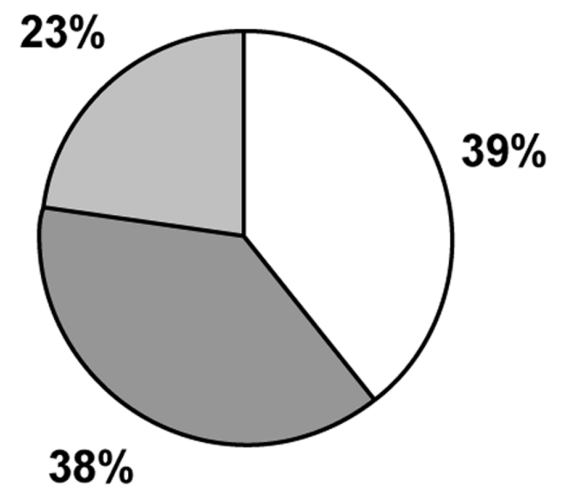

$\square$ Tradizionale $\square$ Aspirazione $\square$ Getto d'acqua

Figura 1. Percentuale di contaminazione raggruppata secondo il metodo di eliminazione del materiale specifico a rischio.

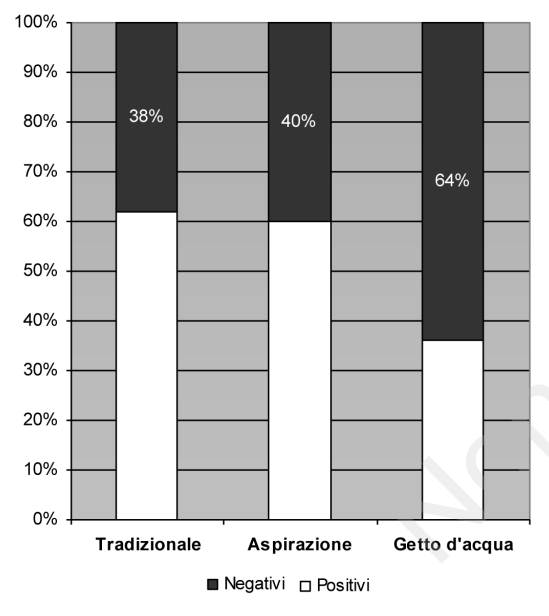

Figura 2. Percentuali di contaminazione rilevate per ognuno dei tre metodi.

\section{Risultati}

La Figura 1 mostra la percentuale di contaminazione raggruppata secondo il metodo di eliminazione del MSR. In Tabella 1 sono riportati i risultati dell'analisi statistica effettuata per valutare la differenza fra i tre metodi di rimozione del midollo spinale. Le differenze di contaminazione fra i tre metodi sono significative $(\mathrm{P}=0,005)$, e la tecnica mediante getto d'acqua è risultata essere quella che garantisce il livello più basso di contaminazione. Nella Figura 2 sono riportate le percentuali di contaminazione rilevate per ognuna delle tre metodiche. Per quanto riguarda la valutazione della correlazione fra lo stato di pulizia della sega circolare e la contaminazione da MSR (studio esplorativo), l'analisi condotta con il test per dati non parametrici della somma dei ranghi di Wilcoxon mostra una differenza significativa tra i campioni eseguiti con la sega pulita rispetto a quelli con sega sporca $(\mathrm{P}=0,015)$.

\section{Conclusioni}

Le metodiche alternative garantiscono una riduzione della contaminazione da MSR. La tecnica mediante aspirazione necessita di ulteriore implementazione in quanto non permette la completa eliminazione del midollo spinale. La metodica per idropulsione garantisce una completa e rapida eliminazione del MSR e qualora applicata in routine garantirebbe pertanto una sensibile riduzione dell'esposizione del consumatore al rischio BSE. La contaminazione residua dei campioni ottenuti mediante tale metodica sembra effettivamente imputabile a una cross-contaminazione operata della sega circolare precedentemente utilizzata nell'ambito della macellazione tradizionale, come peraltro evidenziato da altri autori (Helps et al., 2002; Troeger, 2004).

\section{Bibliografia}

Bowling MB, Belk KE, Nightingale KK, Goodridge LD, Scanga JA, Sofos JN, Tatum JD, Smith GC, 2007. Central nervous system tissue in meat products: an evaluation of risk, prevention strategies, and testing procedures. Adv Food Nutr Res 53:39-64.

Commissione Europea, 2001. Decisione del 22 maggio 2001 recante disposizioni per la prevenzione, il controllo e l'eradicazione di alcune encefalopatie spongiformi trasmissibili, 999/2001/CE. In: Gazzetta Ufficiale, L 147/1, 31/05/2001.

Helps CR, Hindell P, Hillman TJ, Fisher AV, Anil H, Knight AC, Whyte RT, O'Niell DH, Knowles TG, Harbour DA, 2002. Contamination of beef carcasses by spinal cord tissue during splitting. Food Control 13:417-23.

Piscopo A, 2001. Tecnica di rimozione del midollo spinale. Il Progresso Veterinario 7:354-6.

Prendergast DM, Sheridan JJ, Daly DJ, McDowell DA, Blair IS, 2003. Dissemination of central nervous system tissue from the brain and spinal cord of cattle after captive bolt stunning and carcass splitting. Meat Sci 65:1201-9.

Ramantanis SB, 2006. Alternative cattle slaughtering technologies and/or measures reducing the dissemination of central nervous system tissue during head handling, harvesting of cheek meat and tongue and carcass splitting - a review. Vet Arhiv 76:19-36.

Troeger K, 2004. Overview of current and alternative slaughter practices. Biotechnol Agron Soc Environ 8:275-81. 